\title{
WEE1 kinase inhibitor shows promise
}

Patients with locally advanced pancreatic cancer often receive gemcitabine plus radiotherapy. Despite some clinical benefit, virtually all patients have disease progression, indicating a need for more effective therapies. Now, data from a single-arm, dose-escalation phase I trial demonstrate prolonged overall survival (OS) with the addition of the Weel kinase inhibitor adavosertib to gemcitabine plus radiotherapy, relative to historical cohorts.

A total of 34 patients received gemcitabine on days 1 and 8 of a 3-week cycle, followed by adavosertib 3-4 and 24 hours after each dose of gemcitabine, for a maximum of 8 cycles with image-guided, intensity-modulated radiation therapy delivered during cycles 2 and 3 . The adavosertib dose was determined on the basis of the response of the previous patient using the TITE-CRM algorithm. Determining an optimal target dose and toxicity profile were the primary end points of this study.

Dose-limiting toxicities, including anorexia, nausea, fatigue, abdominal pain, altered mental status, liver enzyme elevation and neutropenic fever or thrombocytopenia were observed in eight patients (24\%); $150 \mathrm{mg}$ (dose level 1) was selected as the optimal dose for phase II testing. An analysis of hair follicles in skin biopsy samples revealed phospho-CDK1 suppression, suggesting on-target activity of adavosertib in 16 of 20 consenting patients.

The median OS duration among 20 patients who received any dose of adavosertib was 21.7 months, with a median progression-free survival duration of 9.5 months. As noted by the authors, these data compare favourably to results obtained with gemcitabine plus radiotherapy in previous studies (median OS 11-15 months).

Despite several limitations (among others, use of a single-centre design and a small cohort size), these findings seem promising; data from phase II trials, with larger cohorts of patients, are eagerly awaited.

Peter Sidaway

ORIGINAL ARTICLE Cuneo, K. C. et al. Dose escalation trial of the Wee1 inhibitor adavosertib (AZD1775) in combination with gemcitabine and radiation for patients with locally advanced pancreatic cancer.J. Clin. Oncol. https://doi.org/ 10.1200/JCO.19.00730 (2019)

\section{A new standard for EGFR-mutant NSCLC?}

Over the past decade, EGFR inhibition has supplanted chemotherapy as the standard first-line treatment of advanced-stage EGFR-mutant non-small-cell lung cancer (NSCLC). However, new findings might prompt renewed use of chemotherapy in this setting.

In an open-label, single-centre phase III trial involving 350 patients with untreated advanced-stage EGFR-mutant NSCLC, the addition of carboplatin and pemetrexed to the first-generation EGFR inhibitor gefitinib resulted in improvements in the objective response rate ( $75 \%$ versus $63 \%$ with gefitinib alone; $P=0.01$ ) and the primary end point, progression-free survival (PFS; median 16 months versus 8 months; HR 0.51, 95\% Cl 0.39-0.66; $P<0.001$ ), with consistent benefit across exploratory patient subgroups. These outcomes are similar to those achieved with the third-generation EGFR inhibitor osimertinib as monotherapy in the FLAURA trial. Interestingly, however, $21 \%$ of patients in this trial had a performance status (PS) of 2, whereas all those in FLAURA had a PS $\leq 1$.

In exploratory analyses, the combination therapy was also associated with a prolonged PFS2 (time from randomization to second progression): median 23 months versus 14 months (HR 0.69, 95\% Cl 0.53-0.92; $P<0.001$ ). Accordingly, at a median follow-up duration of 17 months, the median overall survival (OS) duration was not reached versus 17 months (HR 0.45, 95\% Cl 0.31-0.65; $P<0.001$ ); the estimated 18 -month OS was $74 \%$ versus $49 \%$.

Unsurprisingly, the combination regimen was more toxic than gefitinib alone (grade $\geq 3$ adverse event rate of $75 \%$ versus $49 \%$ ), owing mainly to increased myelosuppression, nephrotoxicity and hypokalaemia. Toxicities were, however, mostly manageable.

These results confirm the benefits demonstrated in randomized phase II trials combining gefitinib with carboplatin and pemetrexed, presenting a new first-line treatment option. Whether such regimens will supersede frontline EGFR inhibitor monotherapy remains to be seen, particularly in light of the imminent and reportedly positive OS results of FLAURA.

David Killock

ORIGINAL ARTICLE Noronha, V. et al. Gefitinib versus gefitinib plus pemetrexed and carboplatin chemotherapy in EGFR-mutated lung cancer. J. Clin. Oncol. https://doi.org/ 10.1200/JCO.19.01154 (2019)

\section{BREAST CANCER}

Personalized MRD assays and therapy?

Assessment of molecular minimal residual disease (MRD) could potentially be leveraged, for example, to avoid overtreatment of early stage breast cancer through individualization of local and/or adjuvant therapy based on response to neoadjuvant therapy (NAT). In this context, however, $>90 \%$ of women have no MRD detected using current liquid biopsy assays, regardless of response to NAT, thus precluding reliable risk stratification. The newly developed targeted digital sequencing (TARDIS) platform for personalized detection and quantitation of circulating tumour DNA (ctDNA) might have the required sensitivity.

Combining the strengths of PCR and ligation-based sequencing assays, TARDIS enables screening of plasma cell-free DNA for several patient-specific founder mutations stringently identified via whole-exome sequencing of tumour DNA. After extensive technical validation of TARDIS using reference samples - revealing a sensitivity of at least $53.1 \%$ at a variant allele fraction (VAF) of $0.003 \%$ in a clinically feasible volume of plasma $(4 \mathrm{ml})$, with almost $100 \%$ specificity the assay was applied to 80 plasma samples from 33 women with stage I-III breast cancer, with 6 to 115 mutations assessed per patient.

ctDNA was detected in pretreatment samples from all but one patient, at a median VAF of $0.11 \%$, and in post-NAT samples from 17 of 22 women, at a median VAF of $0.016 \%$ a median $84 \%$ decrease. Notably, 5 of 9 women with a pathological complete response (pCR) had MRD detected, compared to 12 of 13 without a pCR. Moreover, the ctDNA AFs were almost sixfold lower in those with a pCR (median $0.003 \%$ versus $0.018 \%$ ); the median decrease in ctDNA concentrations was $96 \%$ versus $77 \%$. Accordingly, ctDNA fraction enabled prediction of pathological residual disease with an area under the curve of 0.83 .

Importantly, development of the patientspecific assays typically took 3-4 weeks from the time of diagnostic biopsy sampling, which is compatible with the timeframe of post-NAT decision-making. The authors acknowledge, however, that prospective studies with larger cohorts and long-term follow up are needed to establish the optimal diagnostic thresholds and the clinical validity and utility of TARDIS.

\section{David Killock}

ORIGINAL ARTICLE McDonald, B. R. et al. Personalized circulating tumor DNA analysis to detect residual disease after neoadjuvant therapy in breast cancer. Sci. Transl Med. 11, eaax7392 (2019) 